\title{
PRIMARY SARCOMAS OF THE MEDIASTINUM: RESULTS OF THERAPY
}

Michael Burt, MD, $\mathrm{PhD}^{\mathrm{a}}+$

Janet K. Ihde, $\mathrm{MD}^{\mathrm{a} *}$

Steven I. Hajdu, MD $^{\mathrm{b}}$

James W. Smith, MD ${ }^{a * *}$

Manjit S. Bains, MD

Robert Downey, $\mathrm{MD}^{\mathrm{a}}$

Nael Martini, MD

Valerie W. Rusch, MD ${ }^{\mathrm{a}}$

Robert J. Ginsberg, MD
Primary sarcomas of the mediastinum are rare, and data concerning treatment and results of therapy are sparse. Objective: To assess presentation, management, prognostic factors, and survival in mediastinal sarcomas. Methods: We reviewed our experience with 47 patients with the diagnosis of primary sarcoma of the mediastinum. Data were collected from a computerized institutional database and medical records. Survival was analyzed by Kaplan-Meier method and comparisons of survival by log rank test. Results: The median age of 47 patients with mediastinal sarcoma was 39 years (range 2.5 to 69 years), with a male/female ratio of 1.6. The most common complaints were chest/shoulder pain (38\%) and dyspnea $(23 \%)$. The most common tumor types were malignant peripheral nerve tumor (26\%), spindle cell sarcoma (15\%), leiomyosarcoma (9\%), and liposarcoma (9\%). Operation was the primary treatment modality in $72 \%$ of cases $(n=$ 34); 22 sarcomas (47\%) were completely resected. The overall 5-year survival was $32 \%$. High-grade lesions had a significantly decreased survival (5-year survival $=27 \%$ ) compared with low-grade tumors (5-year survival $=66 \%)(p=0.05)$. The overwhelming factor determining survival was the ability to completely resect the tumors (5-year survival $49 \%$ for complete resection; 3-year survival $18 \%$ for incomplete or no resection) $(p=0.0016)$. Despite complete resection, local recurrence occurred in $64 \%$ of cases. Conclusion: Because the overall survival for patients with mediastinal sarcomas is $32 \%$ and the local recurrence is $64 \%$ for tumors completely resected, aggressive adjuvant therapy should continue to be systematically explored. (J Thorac Cardiovasc Surg 1998;115:671-80)
S arcomas originating in the mediastinum are rare, and the literature has primarily reflected anecdotal case reports. No consistent approach in the diagnosis and management of these tumors has been described. Like sarcomas originating in the retroperitoneum, most cases have presented as large

From The Thoracic Service, Department of Surgery and the Pathology Department, ${ }^{\text {b }}$ Memorial Sloan-Kettering Cancer Center, New York, N.Y.

Received for publication March 17, 1997; revisions requested July 15, 1997; revisions received Sept. 26, 1997; accepted for publication Sept. 29, 1997.

Address for reprints: Robert J. Ginsberg, MD, Memorial SloanKettering Cancer Center, 1275 York Ave., New York, NY 10021.

* Present address: Desert Hospital Comprehensive Cancer Center, 1695 N. Sunrise Way, Palm Springs, CA 92262.

**Present address: Department of Surgery, Division of Surgical Oncology, Loma Linda University Medical Center, Loma Linda, CA 92354.

$\dagger$ Deceased.

Copyright (C) 1998 by Mosby, Inc.

$0022-5223 / 98 \$ 5.00+0 \quad \mathbf{1 2 / 1 / 8 6 5 2 5}$ tumors close to vital structures, making management difficult. Because of the paucity of information on presentation, treatment, and outcome in patients with primary sarcomas of the mediastinum, we reviewed our experience at Memorial Sloan-Kettering Cancer Center.

\section{Materials and methods}

A computerized search of the medical records at our institution from January 1, 1940, to December 31, 1991, yielded charts of 47 patients with primary mediastinal sarcoma. Primary mediastinal sarcoma was defined as a sarcoma arising in the anatomic space between the two pleurae of the chest. The anterior mediastinum included the area bounded by the undersurface of the sternum and the anterior surfaces of the great vessels. The middle mediastinum, which is also known as the visceral compartment, was depicted as the area bounded anteriorly by the anterior compartment and extending to the anterior longitudinal spinal ligament; it included sarcomas surrounding the pericardium, trachea, and main bronchi and surrounding or originating from the heart. The posterior mediastinum was defined as the costovertebral region or paravertebral sulci. ${ }^{1}$ Seventy-nine percent of the patients 
Table I. Primary sarcoma of the mediastinum: Clinical presentation

\begin{tabular}{lrr}
\hline & $n$ & $\%$ \\
\hline Complaints & 18 & 38 \\
Chest/shoulder pain & 11 & 23 \\
Dyspnea & 4 & 9 \\
Cough & 4 & 9 \\
Paresthesias & 3 & 6 \\
Hemoptysis & 3 & 6 \\
Fever of unknown origin & 2 & 4 \\
Hoarseness & 1 & 2 \\
Bilateral arm phlebitis & 1 & 2 \\
Horner's syndrome & 1 & 2 \\
Acute myocardial infarction & 9 & 19 \\
Weight loss & & \\
Past history & 5 & 11 \\
Previous radiation to chest & 3 & 9 \\
von Recklinghausen's disease & 1 & 2 \\
Retinoblastoma & &
\end{tabular}

(37 of 47) had tissue available for review, and the slides were reexamined by one pathologist (S.I.H.), who classified tumors as low or high grade on the basis of cellular differentiation, degree of tumor cellularity and vascularity, amount of necrosis, and the number of mitoses per 10 high-power microscopic fields., ${ }^{2,3}$ We performed a detailed retrospective review of the medical records with emphasis on pertinent past medical history, presentation, diagnostic evaluation, histology, treatment, and outcome. We defined local recurrence as the new appearance of sarcoma in the site of definitive therapy. Metastases were defined as sarcoma distinctly removed from the site of the primary tumor. Actuarial survival was calculated by the Kaplan-Meier method and differences in survival were determined by log-rank analysis. ${ }^{4}$

\section{Results}

Clinical presentation. Of the 47 patients who were seen at Memorial Sloan-Kettering Cancer Center with a diagnosis of mediastinal sarcoma, 29 were male and 18 were female (ratio 1.6 to 1 ). The median age was 39 years (range 2.5 to 69 years). Three patients were 11 years old or younger; excluding these three, the median age was 41 years (range 18 to 69 years).

Six patients $(13 \%)$ had a history of radiation therapy 6 to 21 years before diagnosis of mediastinal sarcoma. One patient had a mediastinal liposarcoma develop 16 years after radiation therapy for retinoblastoma. Three patients received mantle radiation for Hodgkin's disease; two had malignant peripheral nerve tumors, and one had an unclassified highgrade sarcoma. Two received mediastinal radiation for non-Hodgkin's lymphoma; one had a spindle cell sarcoma, the other a rhabdomyosarcoma. Three patients had von Recklinghausen's disease. Two of these had malignant peripheral nerve tumors and one a high-grade leiomyosarcoma.

Forty-two patients had a documented period of symptoms, the median of which was 4 months; ranging from 0 to 16 months. Presenting complaints included chest or shoulder pain in 18 patients (38\%), dyspnea in 11 patients (23\%), cough in 4 patients $(9 \%)$, paresthesias in 4 patients $(9 \%)$, hemoptysis in 3 patients $(6 \%)$, fever of undetermined origin in 3 patients $(6 \%)$, and hoarseness in 2 patients $(4 \%)$.

Bilateral upper extremity thrombophlebitis, Horner's syndrome, and acute myocardial infarction were seen in one patient each. Weight loss was noted in nine patients (19\%). In these patients the average reported weight loss was 15 pounds (Table I).

Method of diagnosis. The presence of a mediastinal tumor was most often documented by chest x-ray film. Twenty-five patients had only a chest $x$-ray film without other diagnostic studies before undergoing exploratory thoracotomy. In more recent years computerized tomography (13 patients) and magnetic resonance imaging (six patients) have been used in addition to plain chest films.

Of the 41 patients who had the location of the primary tumor recorded, $17(41 \%)$ were noted to be in the anterior mediastinal compartment and 20 $(49 \%)$ were in the posterior mediastinum. Four patients $(10 \%)$ had middle mediastinal sarcomas, including two originating from the heart (Fig. 1). Clinical size of the lesions was recorded in 21 patients, and ranged from 4 to $20 \mathrm{~cm}$, with a median of $10 \mathrm{~cm}$. The estimated clinical size of the sarcomas correlated closely with the pathologic specimen measurements.

Histology and grade. The histology and grade of the 47 primary sarcomas of the mediastinum are listed in Table II. The most common types of sarcoma in this series were malignant peripheral nerve tumor (MPNT) $(n=12,26 \%)$, spindle cell sarcoma $(n=7,15 \%)$, leiomyosarcoma $(n=5$, $11 \%)$, embryonal rhabdomyosarcoma $(n=4,9 \%)$, and liposarcoma $(n=4,9 \%)$. Of the tumors able to be graded, 25 patients (68\%) had high-grade and 12 patients (32\%) had low-grade tumors; 10 patients (21\%) had insufficient material for grading by our reviewing pathologist. Of the 12 MPNTs, 4 were noted to be high grade, 5 were low grade, and 3 were ungraded. Of the undifferentiated spindle cell sarcomas, three were high grade, one was low grade, and three were not graded. All five leiomyosarcomas 


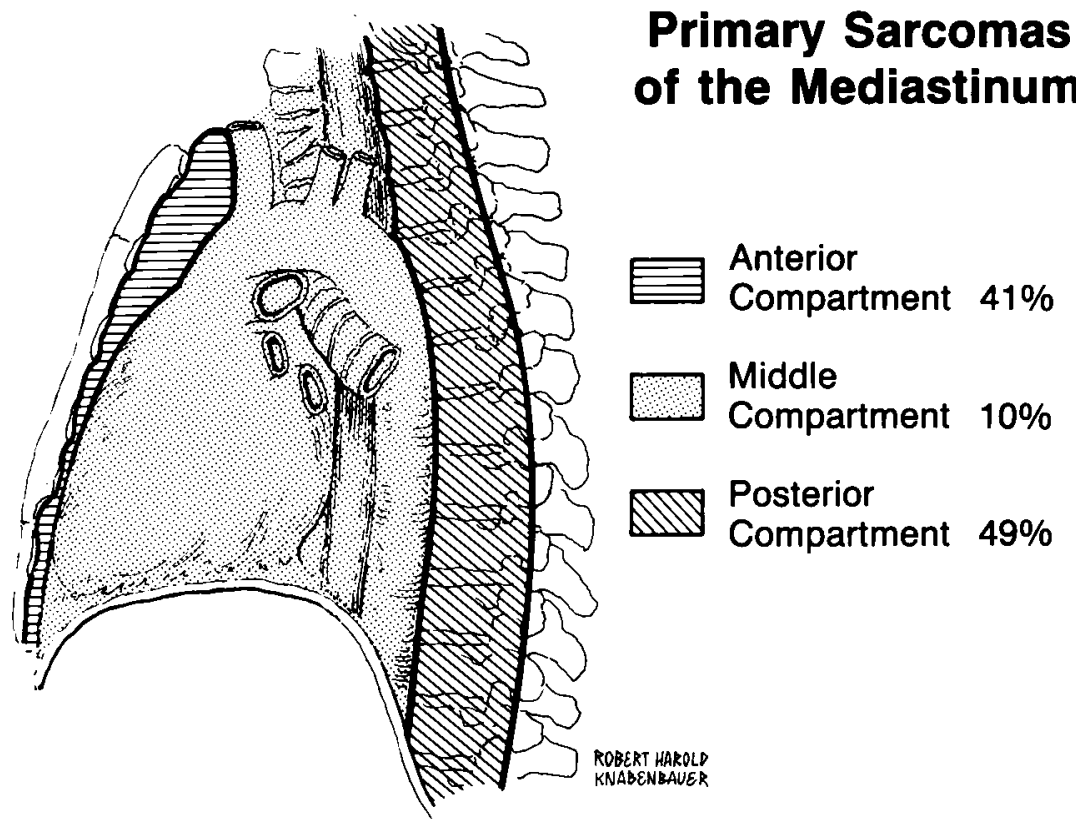

Fig. 1. Location of the primary mediastinal sarcoma was recorded in 41 patients. Seventeen were located in the anterior compartment, 4 in the middle compartment, and 20 in the posterior compartment.

Table II. Primary sarcoma of the mediastinum: Histologic findings and grade

\begin{tabular}{|c|c|c|c|c|c|}
\hline & $n$ & $\%$ & $\begin{array}{c}\text { High } \\
\text { grade } \\
(n)\end{array}$ & $\begin{array}{c}\text { Low } \\
\text { grade } \\
(n)\end{array}$ & $\begin{array}{c}\text { Ungraded } \\
\text { (n) }\end{array}$ \\
\hline Malignant peripheral nerve tumor & 12 & 26 & 4 & 5 & 3 \\
\hline Spindle cell sarcoma & 7 & 15 & 3 & 1 & 3 \\
\hline Leiomyosarcoma & 5 & 11 & 5 & 0 & 0 \\
\hline Embryonal rhabdomyosarcoma & 4 & 9 & 3 & 0 & 1 \\
\hline Liposarcoma & 4 & 9 & 2 & 2 & 0 \\
\hline Fibrosarcoma & 3 & 6 & 2 & 1 & 0 \\
\hline Malignant fibrous histiocytoma & 2 & 4 & 2 & 0 & 0 \\
\hline Angiosarcoma & 2 & 4 & 0 & 1 & 1 \\
\hline Rhabdomyosarcoma & 2 & 4 & 1 & 0 & 1 \\
\hline Unclassified & 2 & 4 & 1 & 1 & 0 \\
\hline Chondrosarcoma & 1 & 2 & 0 & 0 & 1 \\
\hline Hemangiopericytoma & 1 & 2 & 0 & 1 & 0 \\
\hline Osteosarcoma (extraosseus) & 1 & 2 & 1 & 0 & 0 \\
\hline Synovial sarcoma & 1 & 2 & 1 & 0 & 0 \\
\hline Totals & 47 & 100 & 25 & 12 & 10 \\
\hline
\end{tabular}

and both malignant fibrous histiocytomas were highgrade tumors. The embryonal rhabdomyosarcomas were noted to be high grade in three, and one was ungraded. The remaining sarcomas were equally divided between high and low grades. Four $(18 \%)$ of the 22 completely resected tumors were noted to have associated lymph node involvement: two spindle cell sarcomas, one embryonal rhabdomyosarcoma, and one angiosarcoma.
Treatment approaches. Operation was the primary treatment modality in $72 \%$ of patients (34 of 47). Fifteen patients received radiation therapy in addition to the operation. Thirteen patients received chemotherapy either before or after operation, and six patients received chemotherapy, radiation, and resection. Eleven patients received chemotherapy and radiation as primary treatment for their tumors. Two patients elected no treatment 
Table III. Primary sarcoma of the mediastinum: Treatment approaches in 47 patients

\begin{tabular}{lr}
\hline \multicolumn{1}{c}{ Primary therapy } & No. \\
\hline None & 2 \\
Surgery only & 0 \\
Surgery and radiation therapy & 15 \\
Surgery and chemotherapy & 13 \\
Surgery, radiation therapy, and chemotherapy & 6 \\
Radiation therapy and chemotherapy & 11 \\
\hline
\end{tabular}

Table IV. Primary sarcoma of the mediastinum: Tumor recurrence

\begin{tabular}{|c|c|c|c|}
\hline & $n$ & Local recurrence & Metastases \\
\hline Completely resected & 22 & $14(64 \%)$ & $7(32 \%)$ \\
\hline \multirow{4}{*}{$\begin{array}{l}\text { Incompletely or not } \\
\text { resected }\end{array}$} & 25 & $=$ & $\underline{13}(52 \%)$ \\
\hline & & 14: 7 high grade & 20: 11 high grade \\
\hline & & 6 low grade & 2 low grade \\
\hline & & 1 ungraded & 7 ungraded \\
\hline
\end{tabular}

after tissue diagnosis (Table III). Of the 30 patients who received chemotherapy, 7 received it preoperatively as "induction" chemotherapy. Most of the chemotherapy regimens included doxorubicin, cyclophosphamide, and/or cisplatin. Nitrogen mustard and ifosfamide were used infrequently. Four patients received primary brachytherapy (intraoperative radiation implants) for suspected or grossly positive resection margins.

Complications of operation. Of the 34 patients who underwent an operative approach to their tumors, 12 $(35 \%)$ had more than one major procedure to treat the primary tumor or recurrence. Five patients underwent three operations, three patients had four operations, and one patient had five operations. Significant complications occurred in 12 patients $(21 \%)$ after a total of 56 operations. These included three patients with pulmonary emboli within 48 hours of operation, upper extremity phlebitis in two, and four cases of pulmonary infection and sepsis. Solitary cases of Brown-Séquard syndrome, major upper gastrointestinal bleeding, and right hand paralysis attest to the gravity of these complications.

Four patients $(12 \%)$ died after operation, two after a complete and two after an incomplete resection. Twenty-two of the 34 patients $(65 \%)$ undergoing operation had a complete resection of the tumor. To achieve a complete resection, common structures resected included chest wall, pericardium, superior vena cava, vertebra, lung, and diaphragm.
Table V. Number of local and distal recurrences in the more common mediastinal sarcomas

\begin{tabular}{lrcc}
\hline \multicolumn{1}{c}{ Sarcoma } & \multicolumn{3}{c}{ Local } \\
& $n$ & recurrence & Metastases \\
\hline Malignant peripheral nerve tumor & 12 & 7 & 4 \\
Spindle cell sarcoma & 7 & 0 & 3 \\
Leiomyosarcoma & 5 & 2 & 4 \\
Embryonal rhabdomyosarcoma & 4 & 1 & 3 \\
Liposarcoma & 4 & 2 & 0 \\
Fibrosarcoma & 3 & 0 & 1 \\
\hline
\end{tabular}

Local recurrence. Of the 47 patients with mediastinal sarcoma, 34 underwent operation, 22 had complete resection, and 12 an incomplete resection. Four of the incompletely resected tumors were believed to have been grossly resected at the time of operation but were later found to have positive margins and were classified as incompletely resected tumors without persistent disease. Fourteen of the 22 completely resected tumors (64\%) recurred locally. Half of the recurrences developed after resection of high-grade lesions (Table IV). Three (14\%) of these patients received preoperative radiation, and seven $(33 \%)$ had postoperative radiation therapy. Ten patients received chemotherapy, including two who had both preoperative and postoperative chemotherapy. Seven of the patients having complete resections with local recurrences underwent chemotherapy and radiation.

Histologically, the most common tumor to recur locally after complete resection was MPNT, which accounted for $50 \%$ of the recurrences in this group (7 of 14). Two of the local recurrences were in liposarcomas, and two occurred after resection of leiomyosarcomas. The remaining locally recurring tumors were single cases of rhabdomyosarcoma, angiosarcoma, and synovial sarcoma (Table V).

Metastases. Twenty patients (43\%) in this series had metastases develop (Table IV). Sixteen had metastases early during various treatments, and four additional patients eventually were diagnosed with metastatic disease subsequent to primary therapy. Of the 22 completely resected tumors, 7 (32\%) patients had metastases develop, including three patients who also had local recurrence. Eleven of the patients with metastatic disease had high-grade lesions (55\%), two had low-grade lesions, and seven had ungraded tumors. Metastases developed in 11 of the 25 high-grade tumors (44\%). Metastases developed in 2 of the 12 low-grade lesions (17\%). Four of five patients with leiomyosarcoma had me- 


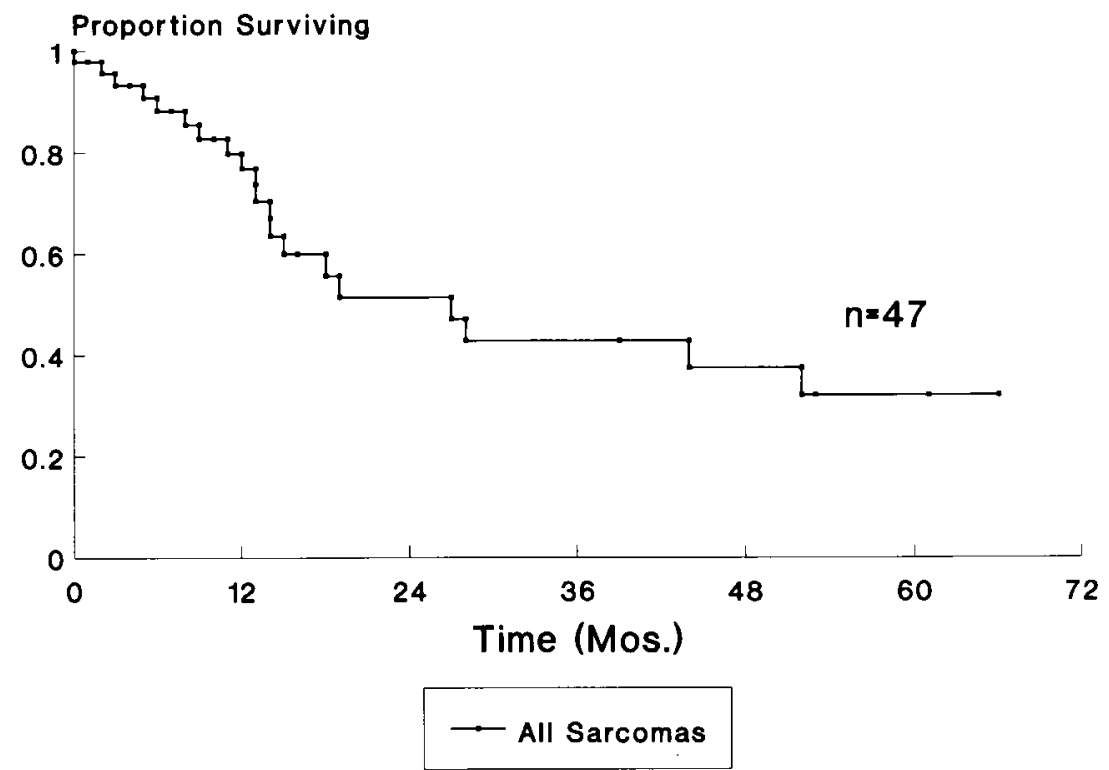

Fig. 2. Overall survival in 47 patients with primary mediastinal sarcoma. Overall actuarial survival was $32 \%$ at 5 years.

tastases develop (80\%). Four of 12 patients $(33 \%)$ with MPNT also had metastases develop, as well as three of four $(75 \%)$ with embryonal rhabdomyosarcomas, and three of seven (43\%) with spindle cell sarcoma eventually had metastases develop (Table V).

Twelve patients had solitary organ metastases only, and eight had multiple organ involvement. The most common site of metastasis was the lung ( 6 of $20,30 \%$ ), followed by liver and bone, each with 5 instances $(25 \%)$. Three patients had brain metastases $(15 \%)$. The subcutaneous tissues and chest wall regions each had two (10\%). Seven of the patients who had metastases received no therapy for their systemic recurrence. Four patients had only chemotherapy and three had only radiation therapy. Two received combination chemotherapy and radiation. Three patients had operations for their metastatic disease and one was operated on in conjunction with radiation and chemotherapy.

Disease-free interval. Of the 22 patients who underwent complete resection of their lesions, 14 had local recurrence (64\%) during or after treatment. Local recurrence occurred in less than 1 month in one patient. Median time to local recurrence was 11 months (range 2 weeks to 132 months). In the seven patients who experienced subsequent metastases after complete resection (including three who also had local recurrence), the median time to diagnosis of distant disease was 14 months (range 2 weeks to 25 months). Of the 12 patients with low-grade tumors, median survival was 3 months after diagnosis of recurrent disease, which was also the median survival in the 25 patients with highgrade tumors after recurrent disease was diagnosed.

Survival. Median follow-up time in this series of 47 patients was 14 months. Median survival was 13 months. Overall survival was $32 \%$ at 5 years (Fig. 2). Patients with high-grade lesions had a significantly decreased survival compared with patients with lowgrade lesions $(p=0.05)$ (Fig. 3). The 5-year survival for patients with high-grade lesions was $27 \%$, and the 5-year survival for the 12 patients with low-grade tumors was $66 \%$. Twenty-two patients had complete resection of their sarcomas and had a significantly prolonged survival compared with the 25 patients who had incomplete or no resection $(p=0.0016)$ (Fig. 4). The 5-year survival in patients who underwent complete resection was $49 \%$; the 3 -year survival in the incompletely or not resected group was only $18 \%$. Grade did not have a significant impact on survival if patients had complete resection $(p=$ 0.12) (Fig. 5). Local recurrence did not gave an impact on survival in the patients who underwent complete resection, although the number of patients who did not experience local recurrence was small $(n=8)$. No difference in survival was found in patients with tumors greater than $10 \mathrm{~cm}$ compared 


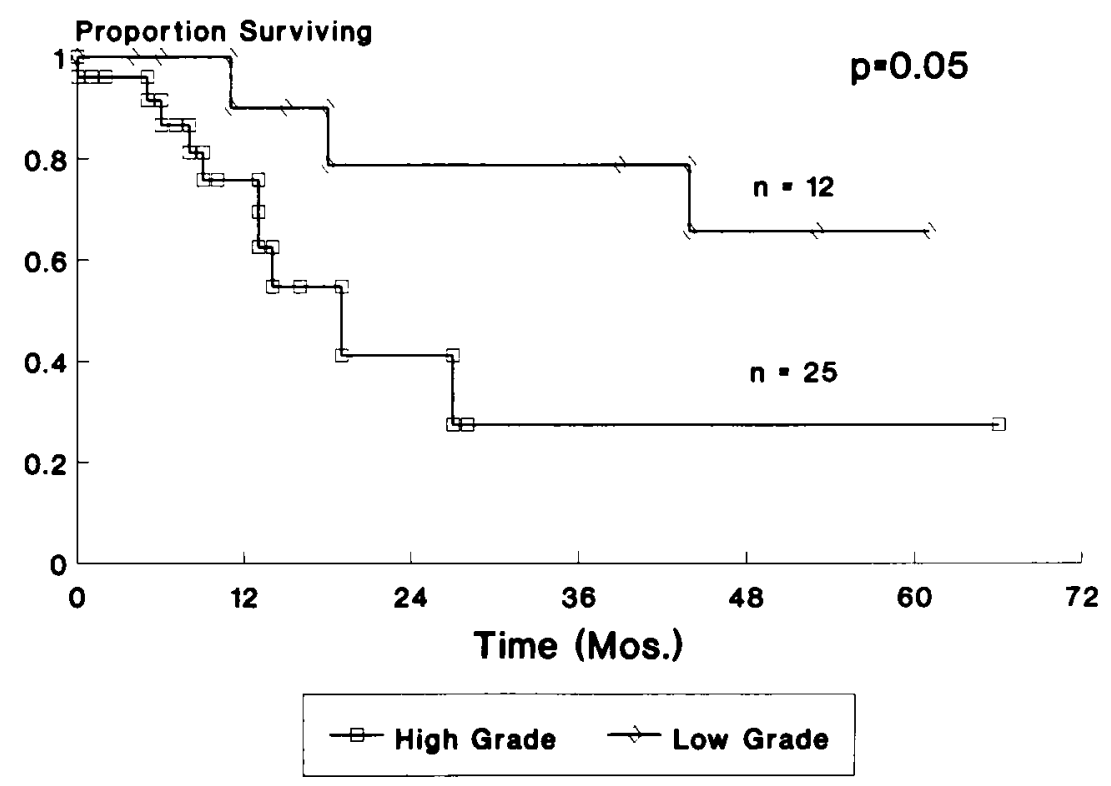

Fig. 3. Overall actuarial survival stratified by tumor grade. Patients with high-grade tumors had a 5-year survival of $27 \%$ compared with the 5-year survival of $66 \%$ in those with low-grade lesions.
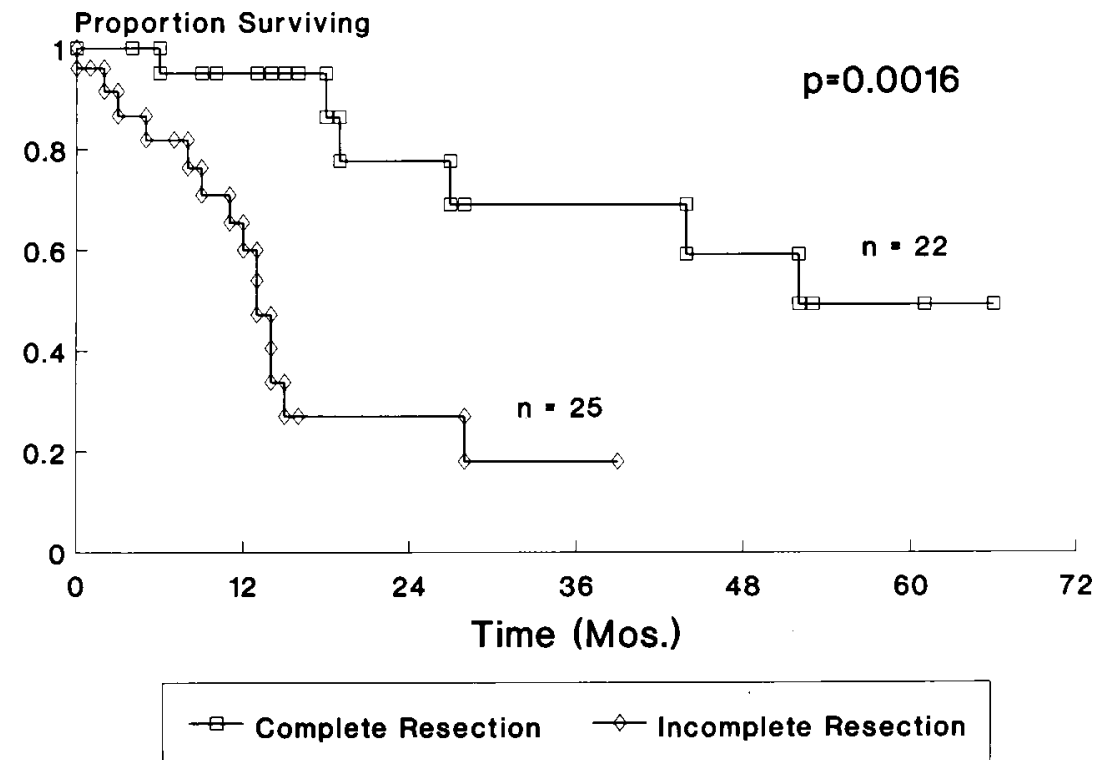

Fig. 4. Actuarial survival curves stratified by completeness of tumor resection demonstrate that those who had complete tumor resection had a 49\% 5-year survival compared with an 18\% 5-year survival in those patients who had incomplete or no resection.

with those with tumors smaller than $10 \mathrm{~cm}$, although a trend $(p=0.08)$ is evident from Fig. 6. We evaluated the influence of age on survival and found no difference in survival in patients less than 39 years old compared with the patients older than 39 .
We examined survival according to histologic findings; however, the number of patients in the different subgroups was too small to allow meaningful analysis. The 12 patients with MPNT histologic findings were noted to have a 5-year survival rate of 


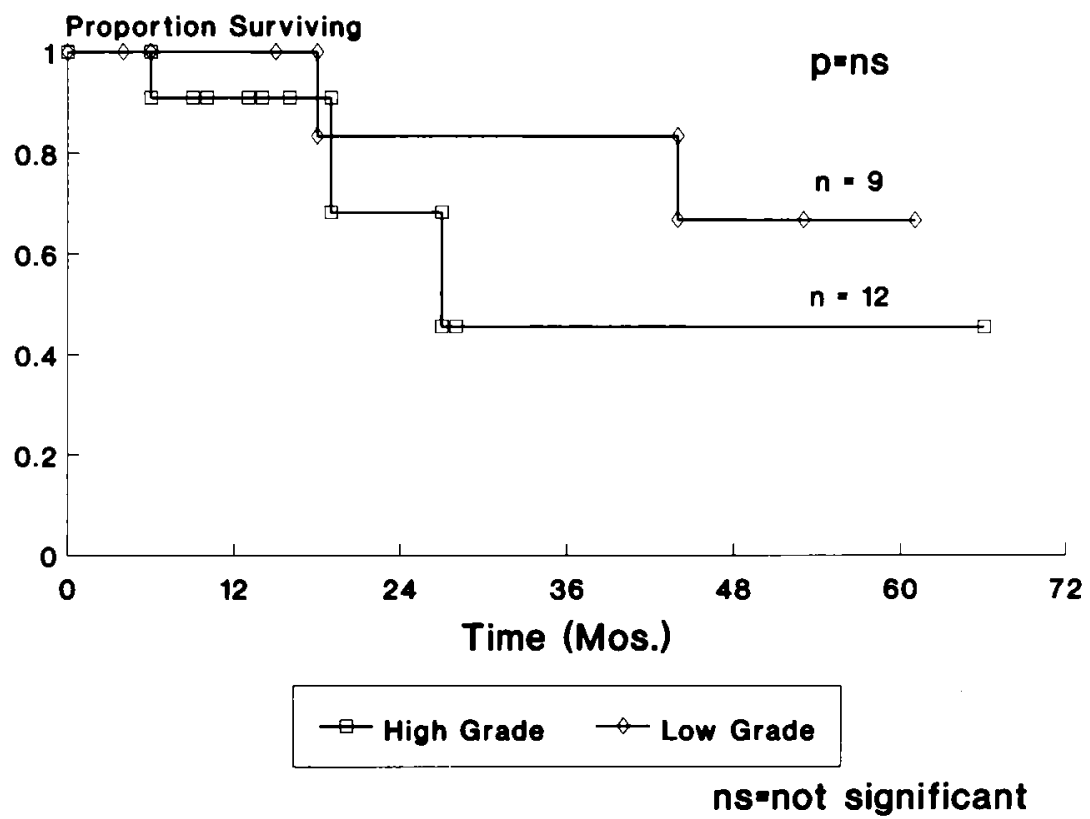

Fig. 5. Overall actuarial survival in patients who had complete resection stratified by grade. In those patients who had complete resection of their sarcomas, tumor grade did not have an impact on survival.

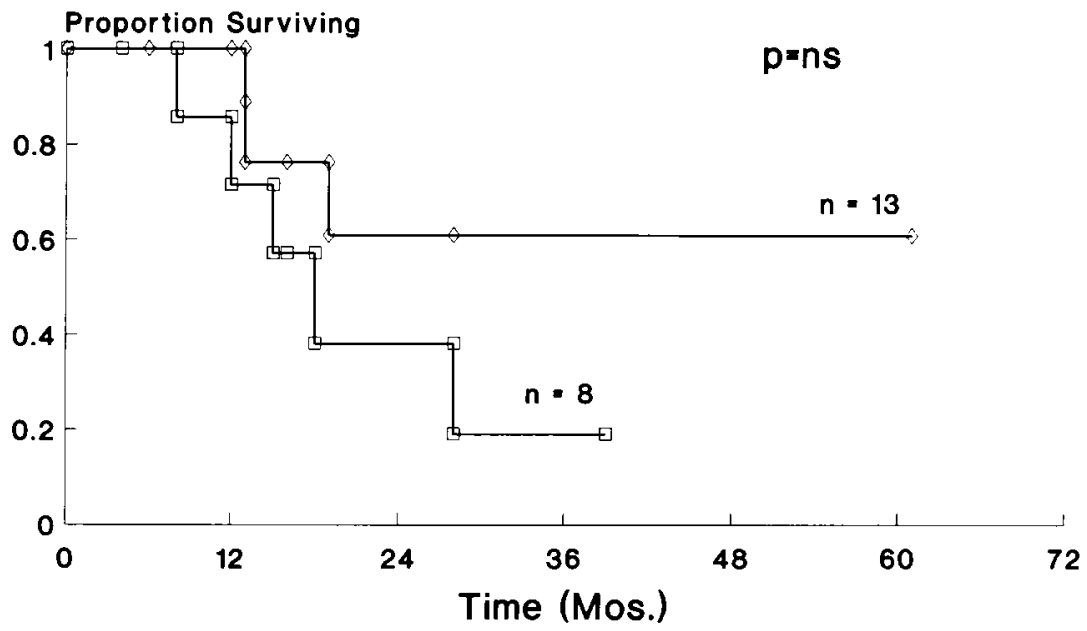

$\multimap<10 \mathrm{~cm} . \quad \longrightarrow, 10 \mathrm{~cm}$.

ns = not significant

Fig. 6. Overall actuarial survival stratified by size in the 21 patients where size had been recorded. The median and mean tumor size was $10 \mathrm{~cm}$. Survival in patients with sarcomas larger than $10 \mathrm{~cm}$ was not significantly different than those with smaller lesions, although a trend is apparent.

$36 \%$, whereas no patient with the other more common sarcomas survived more than 2 years after diagnosis. The impact of the different modalities of treatment was also difficult to analyze because of the wide diversity in treatment approaches. However, it was noted that seven patients completed "induction" chemotherapy before operative resection of their tumors, and four (all high-grade lesions) 
achieved complete tumor resection, two of whom also received radiation therapy.

\section{Discussion}

Primary sarcomas of the mediastinum are rare. Patients with mediastinal sarcomas have a discouragingly poor prognosis despite a multidisciplinary approach. Patients may have ominous signs and/or symptoms because of the location of these lesions. It is also the location of these tumors adjacent to vital structures that makes their management difficult. No consistent approach to the management of patients with primary mediastinal sarcomas has been delineated in the past because of sparse data, as well as the discouraging attempt to control the disease in a hazardous region.

In an attempt to analyze treatment approaches, we retrospectively reviewed clinical presentation, histologic findings, grade, treatment, and outcome in 47 patients who were seen at our institution with a primary mediastinal sarcoma over the past four decades. Because of the rarity of this tumor, our series represents one of the largest. A German series included 22 patients with sarcomas of the mediastinum in 1982, but this series was noted to contain carcinosarcomas, "lymphatic sarcomas," and "round cellular sarcomas," and probably do not represent true sarcomas as currently defined..$^{5}$ To place the incidence of these tumors in perspective, most adolescent and adult mediastinal tumors are benign. Forty percent of mediastinal masses prove to be malignant. ${ }^{6}$ Six percent to $7 \%$ of mediastinal tumors represent mesenchymal neoplasms, and half of these are considered malignant. ${ }^{7}$ In addition, of the neurogenic tumors of the mediastinum, only $10 \%$ to $20 \%$ are estimated to be malignant. ${ }^{6,7}$

A total of 3419 soft tissue sarcomas of all sites was seen at our institution over the past 40 years; our 47 patients with primary mediastinal sarcoma represented $1.4 \%$ of these. Because approximately 6000 sarcomas are seen in the United States yearly, it is estimated that 84 patients per year will have a primary mediastinal sarcoma.

In reviewing clinical presentation of mediastinal sarcomas, we found that most patients are symptomatic. Only five patients (11\%) were asymptomatic and were discovered to have a tumor by incidental chest films. It has been recognized that only $10 \%$ of malignant mediastinal neoplasms are asymptomatic; $90 \%$ of lesions that occur in asymptomatic patients are benign. ${ }^{7}$ The symptoms could be described as nonspecific (chest pain, fever of undetermined ori- gin, weight loss) or caused by compression or invasion of surrounding structures (dyspnea, cough, dysphagia, Horner's syndrome, hoarseness, paresthesia, hemoptysis). Hypoglycemia as a paraneoplastic syndrome has been described in advanced fibrosarcoma, neurofibrosarcoma, and hemangiopericytoma. ${ }^{8,9}$ Pertinent history in a patient with a mediastinal sarcoma includes a history of von Recklinghausen's disease with its propensity to malignant degeneration of neurofibromas. ${ }^{10}$ Four patients $(8.5 \%)$ in our series had this history, of which three had MPNT develop; interestingly, the fourth patient had a leiomyosarcoma.

A history of previous radiation exposure has been recognized as a predisposing factor in the development of sarcomas. ${ }^{11-15}$ In our series three patients had mantle radiation therapy for Hodgkin's disease and two for non-Hodgkin's lymphoma. One patient was irradiated for retinoblastoma. Although radiation-induced sarcomas are more commonly malignant fibrous histiocytomas, none in our series was of this subtype: two were MPNTs, one was a spindle cell sarcoma, one a rhabdomyosarcoma, and one was unclassified. Literature describing radiationinduced sarcomas in the mediastinum is sparse. Most reported cases of sarcoma arising in the field of radiation for Hodgkin's disease or breast cancer are not noted in the mediastinum but rather in the clavicle, scapula, humerus, or subscapular fossa. ${ }^{11,14}$ Sporadic cases in the mediastinum have been reported. ${ }^{12,13}$

As expected, most sarcomas occurred in the posterior mediastinum (49\%), and the most common tumor was MPNT. Nine of $12(75 \%)$ MPNTs occurred in the posterior mediastinum, reflecting the location of major peripheral nerves in the paravertebral sulci. Most authorities have confirmed that the most common tumor in the posterior mediastinum is neurogenic. When tumor is confined to one hemithorax, an intercostal nerve origin is suspected, whereas if the tumor crosses the midline, sympathetic nerve origin is most likely. ${ }^{16}$

The discovery of a mediastinal mass was most often by chest x-ray film in our study. Plain chest films, other than defining the presence of a mass, are not particularly helpful, although the presence of calcification may lead one to suspect chondrosarcoma, osteogenic sarcoma, teratoma, or thymoma. ${ }^{17-19}$ Recently, magnetic resonance imaging has been used to define thoracic malignancies. Magnetic resonance imaging can accurately delineate vascular structures without the use of contrast material and 
seems to better define soft tissue tumors from adjacent normal tissues such as the spinal cord and bone. ${ }^{15,20,21}$ One case report in the literature of a leiomyosarcoma of the superior vena cava was noted to be poorly demarcated by computed tomography and plain chest films; however, the magnetic resonance imaging scan proved useful in defining the location and extent of this tumor. ${ }^{13}$

Tissue obtained by fine-needle aspiration biopsy has been described in the diagnosis of mediastinal seminomas, embryonal carcinomas, carcinoids, and neuroendocrine tumors; however, the usefulness of this technique in the primary diagnosis of sarcomas has not been confirmed, and none of our patients had diagnostic confirmation by this method. ${ }^{22}$

Despite an aggressive operative approach, as well as the use of radiation therapy and/or chemotherapy, the local recurrence rate in patients having a complete resection proved high (64\%), and surpassed the incidence of distant metastasis (43\%). High grade did not contribute toward a higher local recurrence rate. This is in contradistinction to recurrence rates reported for retroperitoneal and visceral sarcomas. ${ }^{23}$ Authorities have agreed that location may prove most important in the frequency of local recurrence in mediastinal sarcomas rather than histologic subtype ${ }^{24}$ and probably reflects the inability to obtain wide margins during resection. Once recurrence has occurred, survival is short (with a median survival of 3 months), despite aggressive resection of recurrences and the use of radiation and chemotherapy. Because of the high incidence of local recurrence of these sarcomas despite complete resection, aggressive adjuvant therapy should continue to be explored.

The grade of primary mediastinal sarcomas had made a significant impact on survival, with highgrade lesions having a poorer prognosis than lowgrade lesions (27\% 5-year survival for high-grade vs $66 \% 5$-year survival for low-grade lesions). However, the factor most predictive of survival was complete resection, and the survival in those completely resected was not influenced by tumor grade. In addition, other factors, such as location and size, must be taken into account. One interesting, but small, subgroup of patients who qualified for complete resection was noted in the seven cases who underwent preoperative chemotherapy. Of these seven, four were completely resected (including two who received adjuvant radiation as well in the form of brachytherapy or postoperative radiation). All four were high-grade tumors. Two patients remain disease-free 13 and 28 months later; two are alive with disease 6 and 66 months later. Because complete resection is the most important prognostic factor determining survival in our series, "induction" chemotherapy may prove to be an important approach.

Survival of patients with soft tissue sarcoma of the chest wall was found to be comparable to that of patients with sarcomas of the extremities. ${ }^{25}$ This may reflect the ability to detect and resect these readily accessible lesions early. Mediastinal sarcomas elude early detection and appear to be similar to retroperitoneal tumors in this respect. Two large series on retroperitoneal sarcomas have also shown that the ability to completely resect these lesions was the most important prognostic factor. The use of radiation therapy and chemotherapy, age, and size did not affect survival, which is similar to the results we describe in this series of mediastinal sarcomas. Unlike our series, grade of the retroperitoneal tumor influenced the survival of patients who had undergone complete resection. ${ }^{26,27}$

Although no consistent approach was used in the management of these tumors, most cases involved multidisciplinary treatments. Despite this, primary sarcomas of the mediastinum have a poor prognosis. The most significant factor having an impact on survival in this series was the ability to completely resect the primary mediastinal sarcoma.

REFERENCES

1. Shields TW. General thoracic surgery. 3rd ed. Philadelphia (PA): Lea \& Febiger; 1989. p. 1251.

2. Hajdu SI. Pathology of soft tissue tumors. Philadelphia: Lea \& Febiger; 1979.

3. Hajdu SI. Differential diagnosis of soft tissue and bone tumors. Philadelphia: Lea \& Febiger; 1986.

4. Kaplan EL, Meier P. Nonparametric estimation from incomplete observations. J Am Stat Assoc 1958;53:457-81.

5. Gebauer C. The postoperative prognosis of primary pulmonary sarcomas. Scand J Thor Cardiovasc Surg 1982;16:91-7.

6. Davis RD Jr, Oldham HN Jr, Sabiston DC Jr. Primary cysts and neoplasms of the mediastinum: recent changes in clinical presentation, methods of diagnosis, management and results. Ann Thorac Surg 1987;44:229-37.

7. Silverman NA, Sabiston DC Jr. Mediastinal masses. Surg Clin North Am 1980;60:757-77.

8. Rosenberg JC. Neoplasms of the mediastinum. In: Devita V, Hellman S, Rosenberg SA, editors. Principles and practice of oncology. 3rd ed. Philadelphia (PA): JB Lippincott; 1989. p. 706-23.

9. Witkin GB, Rosai J. Solitary fibrous tumor of the mediastinum. Am J Surg Pathol 1989;13:547-57.

10. Hajula A, Mattila S, Luosto R, et al. Mediastinal neurogenic tumors: early and late results of surgical treatment. Scand J Thor Cardiovasc Surg 1986;20:115-8. 
11. Hatfield PM, Schulz MD. Postirradiation sarcoma. Radiology 1970;96:593-602.

12. Catanese J, Dutcher JP, Dorfman HD, Andres DF, Wiernik $\mathrm{PH}$. Mediastinal osteosarcoma with extension to lungs in a patient treated for Hodgkin's disease. Cancer 1988;62:2252-7.

13. Weiss KS, Zidar BL, Wang S, et al. Radiation-induced leiomyosarcoma of the great vessels presenting as superior vena cava syndrome. Cancer 1987;60:1238-42.

14. Aprin H, Calandra J, Mir R, Lee JY. Radiation-induced chondrosarcoma of the clavicle complicating Hodgkin's disease. Clin Orthop 1986;209:189-193.

15. Shiu MH, Brennan MF. Clinical features and diagnosis of soft tissue sarcoma. In: Shiu MH, Brennan MF, editors. Surgical management of soft tissue sarcoma. Philadelphia (PA): Lea \& Febiger; 1989. p. 23-38.

16. Prusty S, Bhargava S, Talwar JR, et al. Mediastinal tumors and cysts. Int Surg 1973;58:775-9.

17. Tarr RW, Kerner T, McCook B, Page DL, Nance DP, Kaye JJ. Primary extraosseous osteogenic sarcoma of the mediastinum. South Med J 1988;81:1317-9.

18. Phillips GW, Choong M. Case report: chondrosarcoma presenting as an anterior mediastinal mass. Clin Radiol 1991;43:63-4.

19. Greenwood SM, Meschter SC. Extraskeletal osteogenic sarcoma of the mediastinum. Arch Pathol Lab Med 1989;113: 430-3.
20. Webb WR, Gamou G, Stark DD, et al. Evaluation of magnetic imaging sequences in imaging mediastinal tumors. Am J Radiol 1984;143:525-9.

21. VonShulthess GK, McMurdo K, Tscholakoff D, et al. Mediastinal masses: MR imaging. Radiology 1986;158:289-96.

22. Taccagni G, Cantaboni A, Dell'Antonio G, Vanzulli A, Del Maschio A. Electron microscopy of fine needle aspiration biopsies of mediastinal and paramediastinal lesions. Acta Cytol 1988;32:868-79.

23. Torosian MH, Friedrich C, Godbold J, Hajdu SI, Brennan MF. Soft-tissue sarcoma: initial characteristics and prognostic factors in patients with and without metastatic disease. Semin Surg Oncol 1988;4:13-9.

24. McLean TR, Almassi H, Hackbarth DA, Janjan NA, Potish RA. Mediastinal involvement by myxoid liposarcoma. Ann Thorac Surg 1989;47:920-1.

25. Gordon MS, Hajdu SI, Bains MS, Burt ME. Soft tissue sarcomas of the chest wall. J Thorac Cardiovasc Surg 1991; 101:843-54.

26. Jaques DP, Coit DG, Brennan MF. Soft tissue sarcoma of the retroperitoneum. In: Shiu MH, Brennan MF, editors. Surgical management of soft tissue sarcoma. Philadelphia (PA): Lea \& Febiger; 1989. p. 157-69.

27. Solla JA, Reed KR. Primary retroperitoneal sarcomas. Am J Surg 1986;152:496-8. 